\title{
Breastfeeding and COVID-19 vaccination: position statement of the Italian scientific societies
}

\author{
Riccardo Davanzo ${ }^{1 *}$ (D), Massimo Agosti ${ }^{2}$, Irene Cetin ${ }^{3}$, Antonio Chiantera ${ }^{4}$, Giovanni Corsello ${ }^{5}$, Luca A. Ramenghi ${ }^{6}$, \\ Annamaria Staiano ${ }^{7}$, Marcello Tavio ${ }^{8}$, Alberto Villani ${ }^{9}$, Elsa Viora ${ }^{10}$ and Fabio Mosca ${ }^{11}$
}

\begin{abstract}
The availability of a COVID-19 vaccine has raised the issue of its compatibility with breastfeeding. Consequently, the Italian Society of Neonatology (SIN), the Italian Society of Pediatrics (SIP), the Italian Society of Perinatal Medicine (SIMP), the Italian Society of Obstetrics and Gynecology (SIGO), the Italian Association of Hospital ObstetriciansGynecologists (AOGOI) and the Italian Society of Infectious and Tropical Diseases (SIMIT) have made an ad hoc consensus statement. Currently, knowledge regarding the administration of COVID-19 vaccine to the breastfeeding mother is limited. Nevertheless, as health benefits of breastfeeding are well demonstrated and since biological plausibility suggests that the health risk for the nursed infant is unlikely, Italian scientific societies conclude that COVID-19 vaccination is compatible with breastfeeding.
\end{abstract}

Keywords: Breastfeeding, Vaccination, COVID-19

\section{Background: pandemic and vaccine}

The COVID-19 pandemic was declared by the WHO on 11 March 2020 and Italy was the first country after China to be affected, counting, on 21 December 2020, 1.952.305 cases and 66.717 deaths [1].

During 2020, a huge scientific, organizational and economic effort has led to the availability of vaccines directed against SARS-CoV-2. Despite the acceleration of development, evaluation and approval times, these vaccines are effective, safe and of good quality to fight the pandemic. In Europe, four vaccines will come into use after approval by the European Medicines Agency (EMA): 2 mRNA vaccines containing genetic instructions to induce an immune response against coronavirus (BNT162b2; mRNA-1273) and 2 viral vector vaccines

\footnotetext{
* Correspondence: riccardo.davanzo@gmail.com

${ }^{1}$ Institute for Maternal and Child Health - IRCCS Burlo Garofolo, Via dell'Istria 65/1, 34100 Trieste, Italy

Full list of author information is available at the end of the article
}

using harmless adenoviruses (ChAdOx1-SARS-CoV-2; Ad26.COV2.S) [2].

The public health strategies currently implemented at the international level imply the administration of the vaccine primarily to groups of population most at risk such as healthcare professionals and individuals aged 65 and over. The severity of the pandemic, however, led to shorten the development and evaluation period of COVID-19 vaccines and suggested an emergency regulatory authorization procedure. This acceleration has so far prevented from obtaining enough information on the safety of the COVID-19 among specific population groups such as children and adolescents less than 16 years, pregnant women and nursing women [3].

\section{Compatibility between breastfeeding and COVID- 19 vaccination: indications from non-EU countries}

UK. Initially, breastfeeding women, especially those belonging to the National Health Service (NHS), have been faced with the choice between continuing to breastfeed 
or postponing vaccination ("wait until you have finished breastfeeding before vaccinating yourself") [4]. Instead, the UK Joint Committee on Vaccination and Immunization (JCVI) now advises that although "there is lack of safety data for these specific vaccinations in breastfeeding", there is no known risk in giving these vaccines to breastfeeding women. Ultimately, "breastfeeding women should therefore be offered vaccination if they are otherwise eligible" $[5,6]$.

Canada. The Canadian Pfizer-BioNTech vaccine manufacturer sheet simply points out that it is not known whether the vaccine is excreted in human milk and that a risk to the newborn and infant cannot be excluded [7].

USA. The Food and Drugs Administration (FDA) [8, 9] and the Centers for Disease Control and Prevention (CDC) [10], rather than on the existence of a true contraindication, have emphasized the absence of scientific data: "no data are available to evaluate the effects of the Pfizer-BioNTech COVID-19 vaccine on the breastfed infant or on milk production / excretion". This position de facto leaves open the possibility of breastfeeding women being vaccinated. In fact:

- The US Society for Maternal-Fetal Medicine (SMFM) states that "the vaccine should be offered to women who are breastfeeding" and "are suitable in every other respect" [11];

- The American College of Obstetricians and Gynecologists (ACOG) recommends that COVID19 vaccination also be offered to breastfeeding women, without the need to avoid vaccination or stop breastfeeding [12].

- The Academy of Breastfeeding Medicine (ABM) points out that "it is unlikely that the lipid component of the vaccine enters the bloodstream and reaches the breast tissue. If so, it is even less likely that an intact nanoparticle or mRNA will pass into the milk " [13]. In the unlikely event that mRNA is found in milk, one would expect it to be digested by the baby without having any biological effect. In addition, the ABM notes, vaccination in breastfeeding would allow the passage from mother to infant of specific IgA antibodies against SARSCoV-2 within 5-7 days [2].

EU. On 21 December 2020, the Committee for Medicinal Products for Use in Humans (CHMP) of the European Medicines Agency (EMA) issued a positive opinion on the safety and efficacy of the COVID-19 mRNA (Comirnaty) vaccine in the population aged 16 years and over. The EMA underlines that the vaccine mRNA is rapidly destroyed after the administration of the vaccine. The vaccine can be administered also to those with possible previous asymptomatic SARS-CoV-2 infection and to immunocompromised patients. Although data relating to the use of the vaccine during pregnancy are very limited, actually animal studies do not show dangerous effects. The decision whether or not to vaccinate during pregnancy should be made after consulting health professionals and assessing benefits and risks. Although currently no lactation study is ongoing, according to EMA no specific risk is expected for the breastfeeding mother and her/his nursed infant [14]. Finally, according to the manufacturer sheet, it is not known whether Comirnaty is excreted in human milk [15] and the breastfeeding woman is invited to refer to healthcare personnel. The Italian Medicines Agency has confirmed EMA's indications [16].

\section{Compatibility between breastfeeding and COVID- 19 vaccination: ad interim indications of the Italian scientific societies}

On the basis of the above, a joint ad hoc Working Group of the Italian Society of Neonatology (SIN), the Italian Society of Pediatrics (SIP), the Italian Society of Perinatal Medicine (SIMP), the Italian Society of Obstetrics and Gynecology (SIGO), the Italian Association of Hospital Obstetricians-Gynecologists (AOGOI) and the Italian Society of Infectious and Tropical Diseases (SIMIT) have focused the issue of COVID-19 vaccination while breastfeeding. Between December 14 and 30, 2020 a facilitator gathered the appropriate knowledge, synthetized and balanced the varied opinions and suggestions from the other members of the Working Group. A general unanimous agreement on a preliminary draft was achieved by online communications from the entire panel. Eventually, the final version of the position statement has been approved by the Executive Committees of the adhering Scientific Societies.

Regarding the compatibility between breastfeeding and COVID-19 vaccination, the Italian Scientific Societies affirm that:

- Since breastfeeding must be promoted, protected and supported, due to the positive impact on maternal and child health, society and the environment [17-19], any contraindication to breastfeed should be based on precise medical reasons [20, 21].

- Breastfeeding women should not be systematically invited to stop breastfeeding in order to be vaccinated against COVID-19 [22]. In fact, the decision whether or not to administer the COVID-19 vaccine to the breastfeeding woman should be made after mutual agreement between her and the health professionals, taking into account specific health, social, familiar and work conditions. 
- Biological plausibility suggests that in a breastfed baby the risk resulting from the mother's COVID-19 vaccination is extremely low, while on the other hand, interruption of breastfeeding would lead to a certain loss of its well-documented benefits [23].

- Pregnant and lactating women should be included in future vaccination trials, particularly because pregnancy increases susceptibility to or severity of a disease [24] and because it represents a possible approach to protect the infant in the first months of life, following the transplacental transfer of antiSARS-CoV-2 IgG antibodies [25].

- In conclusion, COVID-19 vaccination is currently considered compatible with breastfeeding.

\section{Abbreviations}

COVID-19: Coronavirus disease 2019; mRNA: Messanger ribonucleic acid

\section{Acknowledgments}

Not applicable.

\section{Authors' contributions}

Riccardo Davanzo conceptualized the position statement and wrote the preliminary draft. All other Authors contributed to drafting and reviewing the manuscript. All Authors read and approved the final version.

\section{Funding}

None to declare

\section{Ethics approval and consent to participate}

Not applicable.

\section{Competing interests}

None of the Authors declares a conflict of interest.

\section{Author details}

${ }^{1}$ Institute for Maternal and Child Health - IRCCS Burlo Garofolo, Via dell'Istria 65/1, 34100 Trieste, Italy. ${ }^{2}$ Woman and Child Department, Ospedale Del Ponte, Varese, Italy. ${ }^{3}$ Department of Woman, Mother and Neonate, Vittore Buzzi Hospital, University of Milan, Milan, Italy. ${ }^{4}$ SIGO. Italian Society of Gynecology and Obstetrics (President), Naples, Italy. ${ }^{5}$ Department of Sciences for Health Promotion and Mother and Child Care "G. D'Alessandro", University of Palermo, Palermo, Italy. 'Department of NeuroscienceRehabilitation-Ophthalmology-Genetics-Child and Maternal Health (DINOGMI), University of Genoa, Genoa, Italy. ${ }^{7}$ Section of Pediatrics, Department of Translational Medical Science, "Federico II" University of Naples, Naples, Italy. ${ }^{8}$ Unit of Emerging and Immunosuppressed Infectious Diseases, Department of Gastroenterology and Transplantation, Azienda Ospedaliero-Universitaria "Ospedali Riuniti", Torrette Ancona, Rome, Italy. ${ }^{9}$ Department of Pediatrics, Bambino Gesù Children's Hospital, Rome, Italy. ${ }^{10} \mathrm{AOGOI}$. Italian Association of Hospital Obstetricians-Gynecologists (President), Turin, Italy. ${ }^{11}$ Department of Clinical Sciences and Community Health, University of Milan, Fondazione IRCCS Cà Granda Ospedale Maggiore Policlinico, Milan, Italy.

Received: 18 January 2021 Accepted: 18 February 2021

Published online: 27 February 2021

\section{References}

1. Epicentro. Sorveglianza integrata COVID-19: i principali dati nazionali. Rome: Istituto Superiore di Sanità. https://www.epicentro.iss.it/coronavirus/sarscov-2-sorveglianza-dati

2. Marco Cavaleri, Biological Health Threats and Vaccines Strategy, EMA. How are COVID-19 vaccines developed? EMA public stakeholder meeting on COVID-19. How safe and effective vaccines are developed and authorised in the EU. 11 December 2020. Virtual Meeting. https://www.ema.europa.eu/en/ human-regulatory/overview/public-health-threats/coronavirus-diseasecovid-19/treatments-vaccines-covid-19

3. Doshi P. Will covid-19 vaccines save lives? Current trials aren't designed to tell us. BMJ. 2020;371:m4037.

4. Public Health England. COVID-19 Vaccination: a guide for women of childbearing age, pregnant, planning a pregnancy or breastfeeding. 6 December 2020. https://www.gov.uk/government/publications/covid-19-va ccination-women-of-childbearing-age-currently-pregnant-planning-a-pregna ncy-or-breastfeeding/covid-19-vaccination-a-guide-for-women-of-childbea ring-age-pregnant-planning-a-pregnancy-or-breastfeeding

5. Medicines \& Health Care Products Regulatory Agency. Regulatory approval of Pfizer/BioNTech vaccine for COVID-19. Information for Healthcare Professionals on Pfizer/BioNTech COVID-19 vaccine. Updated 30 December 2020. https://www.gov.uk/government/publications/regulatory-approval-ofpfizer-biontech-vaccine-for-covid-19

6. Royal College of Obstetricians \& Gynaecologists (RCOG). Update advice on COVID-19 vaccination in pregnancy and women who are breastfeeding. News 30 December 2020. https://www.rcog.org.uk/en/news/updated-a dvice-on-covid-19-vaccination-in-pregnancy-and-women-who-are-brea stfeeding/

7. Pfizer-BioNTech COVID-19 Vaccine (CoVID-19 mRNA Vaccine) Product Monograph (Canada). December 9, 2020.

8. FDA News Release. FDA takes key action in fight against COVID-19 by issuing emergency use authorization for first COVID-19 vaccine. December 11, 2020. https://www.fda.gov/emergency-preparedness-and-response/ coronavirus-disease-2019-covid-19/pfizer-biontech-covid-19-vaccine

9. U.S. Food and Drugs Administration (FDA). Fact Sheet for Health Care providers admministering vaccine (vaccination providers). Emergency use authorization (EUA) of Pfizer-Biontech COVID-19 vaccine to prevent coronavirus disease 2019 (COVID-19). https://www.fda.gov/media/144413/ download

10. Centers fo Disease Control and Prevention (CDC). Vaccine Recommendations and Guidelines of the Advisory Committee on Immunization Practices (ACIP). U.S. Department of Health \& Human Services. USA.gov. 2020. https://www.cdc.gov/vaccines/hcp/acip-recs/vacc-specific/ covid-19/clinical-considerations.html

11. Society for Maternal Fetal Medicine. SMFM Statement: SARS-CoV-2 Vaccination in Pregnancy. SMFM: Provider Considerations for Engaging in COVID-19 Vaccine Counseling With Pregnant and Lactating Patients. 2020 December 1. https://www.smfm.org/publications/search?q\%5Bpublication_ categories_id_eq\%5D=74

12. American College of Obstetricians and Gynecologists (ACOG). Vaccinating pregnant and lactationg patients against COVID-19. December 13, 2020. https://www.acog.org/clinical/clinical-guidance/practice-advisory/articles/202 0/12/vaccinating-pregnant-and-lactating-patients-against-covid-19;

13. Academy of Breastfeeding Medicine (ABM). ABM Statement. Considerattions for COVID-19 vaccination in lactation. December 14. 2020. https://www. bfmed.org/abm-statement-considerations-for-covid-19-vaccination-in-lacta tion?fbclid=|wAR1 qG7rS66lyTDyDlxGIOrwQsoSqDRuXWMWnjgBXwhOJuSofk13veeT3jl8

14. European Medicines Agency. Comirnaty. 21 December 2020. https://www. ema.europa.eu/en/news/ema-recommends-first-covid-19-vaccine-authorisa tion-eu. https://www.ema.europa.eu/en/medicines/human/summariesopinion/comirnaty

15. Comirnaty. Product Information approved by the CHMP on 21 december 2020, pending endorsement by the European Commission.

16. Italian Medicines Agency (AIFA). Vaccinazione anti COVID-19 con vaccino Pfizer mRNABNT162b2 (Comirnaty) FAQ AIFA. Rome; 2021. https://www.aifa. gov.it/documents/20142/1279946/FAQ-Vaccinazione_anti_COVID-19_con_va ccino_Pfizer.pdf/ea9099cd-c71c-d443-e447-6da60137da37

17. Rollins NC, Bhandari N, Hajeebhoy $N$, et al. Why invest, and what it will take to improve breastfeeding practices? Lancet. 2016;387(10017):491-504.

18. Victora CG, Bahl R, Barros AJ, et al. Breastfeeding in the 21st century: epidemiology, mechanisms, and lifelong effect. Lancet. 2016;387(10017): 475-90.

19. Bode L, Raman AS, Murch SH, Rollins NC, Gordon Jl. Understanding the mother-breastmilk-infant "triad". Science. 2020;367(6482):1070-2.

20. World Health Organization (Department of Child and Adolescent Health Development and Department of Nutrition for Health and Development) and UNICEF. Acceptable medical reasons for use of breast-milk substitutes. 
Geneva: WHO; 2009. https://apps.who.int/iris/bitstream/handle/10665/6993 8/WHO_FCH_CAH_09.01_eng.pdf?ua=1

21. Davanzo R. Controversies in Breastfeeding Front Pediatr 2018;6:278. doi: https://doi.org/10.3389/fped.2018.00278. eCollection 2018.

22. Hare H, Womersley K. Healthcare workers who breastfeed should be offered the COVID-19 vaccine. BMJ. 2020:21 https://blogs.bmj.com/bmj/2020/12/21/ healthcare-workers-who-breastfeed-should-be-offered-the-covid-19-vaccine/.

23. Davanzo R, Moro G, Sandri F, Agosti M, Moretti C, Mosca F. Breastfeeding and coronavirus disease-2019: ad interim indications of the Italian Society of Neonatology endorsed by the Union of European Neonatal \& perinatal societies. Matern Child Nutr 2020;16(3):e13010. doi: https://doi.org/10.1111/ mcn.13010. Epub 2020 Apr 26.

24. Zambrano LD, Ellington S, Strid P, et al. Update: Characteristics of Symptomatic Women of Reproductive Age with Laboratory-Confirmed SARS-CoV-2 Infection by Pregnancy Status - United States, January 22 October 3, 2020. MMWR Morb Mortal Wkly Rep. 2020;69:1641-7. https://doi. org/10.15585/mmwr.mm6944e3external.icon.

25. Flannery DD, Gouma S, Dhudasia MB, Mukhopadhyay S, Pfeifer MR, Woodford EC, Triebwasser JE, Gerber JS, Morris JS, Weirick ME, MCAllister CM, Bolton MJ, Arevalo CP, Anderson EM, Goodwin EC, Hensley SE, Puopolo KM. Assessment of Maternal and Neonatal Cord Blood SARS-CoV-2 Antibodies and Placental Transfer Ratios. JAMA Pediatr. 2021: e210038. doi: https://doi.org/10.1001/jamapediatrics.2021.0038. Epub ahead of print. PMID: 33512440; PMCID: PMC7846944.

\section{Publisher's Note}

Springer Nature remains neutral with regard to jurisdictional claims in published maps and institutional affiliations.

Ready to submit your research? Choose BMC and benefit from:

- fast, convenient online submission

- thorough peer review by experienced researchers in your field

- rapid publication on acceptance

- support for research data, including large and complex data types

- gold Open Access which fosters wider collaboration and increased citations

- maximum visibility for your research: over $100 \mathrm{M}$ website views per year

At $\mathrm{BMC}$, research is always in progress.

Learn more biomedcentral.com/submissions 